\title{
On the layout of a least weight single span structure with uniform load
}

\section{Some comments and improvements}

\author{
Jaime Cervera Bravo · Carlos Vázquez Espí · Mariano Vázquez Espí
}

v.2.0 April 28, 2015

\begin{abstract}
Beghini et al (Struct Multidisc Optim doi:10.1007/s00158-013-1030-6, 2013) have published a very interesting paper arriving to practically the same nearly optimal solutions for the so named "bridge problem" that the Writers published a year before, but using an alternative and remarkable approach to the problem. In spite of this general agreement, the Writers think that some details of the paper can be improved and there are results that can be given a clear and meaningful interpretation thanks to an old and practically unknown theorem on optimal slenderness.
\end{abstract}

Keywords Layout optimization · trusses · bridge problem · Optimal Slenderness

\section{Introduction}

The results from Beghini et al (2014) (the Authors hereafter) about nearly optimal layout of single span structure with uniform load, that has a very interesting approach, can be ratified by the Writers and complemented in some details that can be summarized as follows:

- The Writers present here a comparison with very similar results that they have recently published, see

J. Cervera Bravo E-mail: jaime.cervera@upm.es

Universidad Politécnica de Madrid. Escuela Técnica Superior de Arquitectura. Departamento de Estructuras de Edificación. Avda. Juan de Herrera, 4. 28040 Madrid, España.

C. Vázquez Espí E-mail: Carlos.Vazquez.Espi@UPM.es Universidad Politécnica de Madrid. Departamento de Fundamentos Matemáticos. Plaza del Cardenal Cisneros, 3. 28040 Madrid, España.

M. Vázquez Espí E-mail: Mariano.Vazquez.Espi@UPM.es Universidad Politécnica de Madrid. Research Group on Architecture, Urban Planning and Sustainability. Avda. Juan de Herrera, 4. 28040 Madrid, España.
Cervera Bravo et al (2014). These results have been obtained using a different approach on the truss layout definition and optimization that involves variational calculus.

- The application of a theorem on the condition in slenderness for optimality of trusses with vertical loads, stated in nineties by Professor Ricardo Aroca, can easily explain some figures of Authors' work. In particular all cases in their table 1 can be reduced to a single problem through the consideration and comparison between the vertical and horizontal volumes. Their relations extend those presented in the paper among the compression and tension volumes that follow from Maxwell's Lemma. The necessary references, explanation and consequences of this theorem will be fully adressed in section 3 .

Some minor corrections to Authors' paper are also suggested.

\section{Comparison between McConnel, Author's and Writer's solutions for Layouts for Truss-like Bridge Structures}

The approach to the optimization problem followed by the Authors (Beghini et al, 2014), —dealing with the properties of the reciprocal graphs of layout and forcesis a suggestive and promising one. Authors' results can be confirmed from Writers' ones (Cervera Bravo et al, 2014: published online in June 2013), being the latter slighty better. These were obtained using a variational approach on the original truss layout. The comparison of the main figures is summarised in Table 1. The comparison confirms the validity of the Authors simplification for $\theta^{\prime}$, that is $\theta^{\prime}=$ constant, i.e. the imposition of a constant angle change on the arch layout for segments 
Table 1 Comparison of provided values for several layout parameters.

\begin{tabular}{|c|c|c|c|c|c|c|c|c|c|c|c|}
\hline \multirow[b]{2}{*}{ parms. $^{* *}:$} & \multicolumn{2}{|c|}{ McConnel (1974) } & \multicolumn{3}{|c|}{ Authors (eq. 17) } & \multicolumn{3}{|c|}{ Authors (eq. 37) } & \multicolumn{3}{|c|}{ Writers* } \\
\hline & $\hat{y}_{m} / \hat{L}$ & $\nu$ & $\hat{y}_{m} / \hat{L}$ & $\nu$ & $\theta_{1}$ & $\hat{y}_{m} / \hat{L}$ & $\nu$ & $\theta_{1}$ & $\hat{y}_{m} / \hat{L}$ & $\nu$ & $\theta_{1}$ \\
\hline & 0.43 & 0.9899 & 0.4307 & 0.9873 & $-8.68^{\circ}$ & 0.4206 & 0.9861 & $-6.4^{\circ}$ & 0.4419 & 0.9847 & $-4.10^{\circ}$ \\
\hline & 0.33 & 0.8026 & 0.2966 & 0.8138 & $22.04^{\circ}$ & 0.3903 & 0.8026 & $30.2^{\circ}$ & 0.3170 & 0.8010 & $28.92^{\circ}$ \\
\hline & 0.31 & 0.7578 & 0.3188 & 0.7582 & $16.94^{\circ}$ & 0.3165 & 0.7581 & $15.9^{\circ}$ & 0.3617 & 0.7580 & $15.87^{\circ}$ \\
\hline & so & 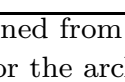 & & & & $r$ & & $\overline{\mathrm{ed}}$ & ry & avo & $(2014)$ \\
\hline
\end{tabular}

between the connections of hangers equally separated on the bridge support line.

It is worth to note that with Writers' approach a closed form for the arch is obtained depending on a sole function, the angle of the hangers with the vertical direction, whereas with Authors' approach the angle used is that of the arch with the vertical (the angle of the spokes in the dual truss with the horizontal); and as the Authors point out they were not able to find a closed, analytical form for the original truss from the closed form of the dual one. It can be concluded that both approaches are not fully equivalent albeit from a practical view their respective results are.

The Writers agree with the Authors about the practical significance of nearly optimal solutions for problems without known absolute optimal solution - or for problems whose optimal solutions are of complex construction - but the search of the absolute optimum is important too as these solutions account for the limit of the economy in each problem and they will serve as a mesure for the quality of nearly optimal solutions. In the bridge problem, the numerical results found up to date (Pichugin et al, 2012; Cervera Bravo et al, 2014; Sokół, 2014) show great evidence for the existence of a Michell optimum in this problem. Nevertheless it must be remembered that the Michell theorem is a "sufficiency" and not a "necessity" condition theorem, and so is the existence of a Michell net, see Michell (1904: pp. 590-591).

\section{On the explanation of some relations between volumes of different but related problems and layouts}

Authors make several remarks about volumes involved in the parabolic arch solution - section 2 on Maxwell theorem and the parabolic arch-. Some of them should be made more precise and, also, similar remarks can be applied to structures not limited to those with a "limiting constant stress (equal in tension and in compression)" if one deals, not with the volume, but with "quantity" of Michell or "stress volume" as shown in Cervera Bravo et al (2014), see Lemma 8 (Michell's Lemma) there. Interesting remarks can be also added trough the application of a theorem from Ricardo Aroca that is explained below.

Let first see some remarks based on Maxwell writings. In the application of Maxwell's Theorem to the parabolic arch - the theorem is also known as Maxwell's Lemma (Cervera Bravo et al, 2014: see Lemma 4)-, Authors assert that $\sum \vec{F} \cdot \vec{r}=0$ for the third case of their Table 1 "since all the loads are applied at the horizontal dashed line" (Beghini et al, 2014:51). This reason is wrong. In fact, this occurs too in the second case but, as Authors correctly say, now $\sum \vec{F} \cdot \vec{r} \neq 0$. It is better to follow Maxwell: the quantity $\sum \vec{F} \cdot \vec{r}$ known as "static constant" or "Maxwell number" (Cervera Bravo et al, 2014: Definition 3) - is the negative of the virtual work done by external forces when the virtual deformation reduces the space to a point. In the third case the key is that since all forces - loads and reactions - are vertical and are applied at the horizontal dashed line (where can be selected the origin for $\vec{r}$ ) the virtual work will be null. In the second case the reactions are not vertical, so there is no orthogonality and the virtual work is not null.

In Maxwell and Michell approaches to structural design there are no mentions to reactions nor displacement conditions, but to sets of equilibrated forces. That has several interesting and fruitful consequences that are discussed on Cervera Bravo et al (2014). 
In the third problem presented by the Authors in their Table 1, Maxwell's Theorem, as Authors correctly say, imposes the identity between compression and tension volumes, as their difference should be null to assure equality to - as the Writers call it- Maxwell number $\mathcal{M}=\sum \vec{F} \cdot \vec{r}=0$. That is true on any solution for the problem, be it optimal or not. So its minimum volume implies the minimum on any of both components and that imposes an identical layout and a half total volume for the pinned arch as Authors' Table 1 shows in its first row - solution from Rozvany and Prager (1979) — due to the full elimination of the tension components, tie and hangers.

\section{Aroca's Theorem on the Optimal Slenderness}

We have seen the significancy of the relations between solutions to first and third problems in Author's Table 1. The interesting part comes now, with the explanation of exact numerical relations between optimal solutions for the second and third problem, that reduce thus all three problems to different forms of a unique problem. In order to do so one must consider Aroca's theorem on the Optimal Slenderness (OST), as Writers remark in Vázquez Espí and Cervera Bravo (2011), paper that Authors cite.

As Professor Ricardo Aroca has not formally published most of its findings, it is not easy to track them: the first published notice of the commented Theorem, that was enuntiated in the first years of nineties in Aroca's doctorate lessons, is in a $\mathrm{PhD}$ Thesis of a disciple (Fernández Cabo, 1998: pp. 30-33 where Author refers to Aroca's doctoral course notes of years 89-90 and 92-93). The theorem can be found in Spanish in Cervera (2010: section 7.2.4). The present discussion is probably the first place were it will be found in its full form in English.

To present, prove and employ the theorem it must be firstly remembered that Authors' volume $V$ as cost measure can be accounted as Michell's quantity (Michell, 1904: p. 590, line 15) $\mathcal{V}=\sum\left|F_{i}\right| L_{i}$ with a price $1 / \sigma$ having $V=\mathcal{V} / \sigma$, or $\mathcal{V}=\sigma V$, as Authors' equations and tables emphasize. The Writers retain $\mathcal{V}$ as the basic magnitude for the structural costs evaluation following Michell's work, under the denomination stress volume. Secondly, as $\mathcal{V}=\sum\left|F_{i}\right| L_{i}=\sum\left|F_{i} L_{i}\right|=\sum \mid \vec{F}_{i}$. $\vec{L}_{i} \mid$ (the scalar product, taking into account that $\vec{F}_{i}$ and $\vec{L}_{i}$ are aligned for any bar $i$ ), that quantity or stress volume can be decomposed into its cartesian components and, in those problems for which all or part of the relevant external forces are parallel, such decomposition can be made in two components, i.e. the parallel direction, and the orthogonal directions, having thus $\mathcal{V}=\mathcal{V}^{\|}+\mathcal{V}^{\perp}$, both for 2D or 3D problems.

That made, given one truss-like structural form for an equilibrated set of parallel external forces, one may consider affine changes in the parallel direction of that layout. That kind of transformations correspond usually to changes in depth in a structural layout sustaining gravity loads with vertical reactions. In those conditions it can be stated:

Theorem 1 (Aroca's Optimal Slenderness Theorem) Let be a given truss solving the problem of balancing an equilbrated system of parallel external forces, loads and reactions. If the layout is subjected to an affine transformation of magnitude $\alpha$ parallel to the forces, which remain unchanged - but can be moved in the transformation with the movement of their application points - the resulting parallel and ortogonal components of the stress volume, $\mathcal{V} \|$ and $\mathcal{V}^{\perp}$ respectively, change accordingly and inversely with $\alpha$, their product remaining constant and equal to the product of $\mathcal{V}_{1}^{\|}$and $\mathcal{V}_{1}^{\perp}$ corresponding to the original layout.

In the former conditions, let consider the set of layouts obtained when the parameter $\alpha$ varies. There exists an optimal layout for which the total stress volume is minimum. It corresponds to

$\mathcal{V}^{\|}=\mathcal{V}^{\perp}=\sqrt{\mathcal{V}_{1}^{\|} \mathcal{V}_{1}^{\perp}}$

the minimum value of the stress volume is

$\mathcal{V}_{\min }=2 \sqrt{\mathcal{V}_{1}^{\|} \mathcal{V}_{1}^{\perp}}$

and the value of the parameter of the affine transformation for which we obtain the optimal layout is

$\alpha^{*}=\sqrt{\frac{\mathcal{V}_{1}^{\perp}}{\mathcal{V}_{1}^{\|}}}$

the slenderness and depth of this optimal layout being

$\lambda^{*}=\frac{1}{\alpha^{*}} \lambda_{1}=\frac{1}{\alpha^{*}} \frac{L}{d_{1}}, \quad d^{*}=\alpha^{*} d_{1}$

where $L$ is the unchanged reference span, and $d_{1}, \lambda_{1}$ are the depth and slenderness of the original layout.

Proof For the shake of brevity let us consider the 2D case. From this the 3D case is an easy task. Let us consider a generic node, $j$, of the truss. Let be $\tilde{F}_{j}^{\|}$and $\tilde{F}_{j}^{\perp}$ the parallel and ortogonal components of the external force applied in this node, which are to be balanced by the internal forces of the bars joined at this node. If the internal force of the bar $i$ over node $j$ is represented by its cartesian components, $F_{i}^{\| l}$ and $F_{i}^{\perp}$, the equilibrium 
equations for the node $j$ when the orthogonal component of the external force $\tilde{F}_{j}^{\perp}$ is null are given by

$$
\begin{aligned}
\tilde{F}_{j}^{\| l} & =\sum_{i} F_{i}^{\|} \\
0 & =\sum_{i} F_{i}^{\perp}
\end{aligned}
$$

where the summation is extended over all the bars joined at the node $j$.

On the other hand, the components of the internal force of the bar $i F_{i}^{\|}$and $F_{i}^{\perp}$ are not independent. Since $F_{i}$ mut be aligned with the length vector $L_{i}$, they should verify the equation

$\frac{F_{i}^{\|}}{F_{i}^{\perp}}=\frac{L_{i}^{\|}}{L_{i}^{\perp}}$

where $L_{i}^{\|}$and $L_{i}^{\perp}$ are the cartesian components of $L_{i}$. This geometrical compatibility equation is written in a more convenient form as

$0=F_{i}^{\| \mid} L_{i}^{\perp}-F_{i}^{\perp} L_{i}^{\|}$

The set of equations (1) and (2) written for all nodes and equations (3) for all bars constitute the set of equations to determine the internal forces in a given layout -and verify both in isostatic as in internally hiperestatic geometries-

Let us consider an affine transformation in depth in the layout, i.e., in the parallel direction, changing $L_{i}^{\|}$ to $L_{i}^{\|}(\alpha)=\alpha L_{i}^{\|}$(and keeping $L_{i}^{\perp}$ in its original value) for all bars.

The position of the nodes are changed accordingly, but the external forces are not changed.

If the truss resulting from the new layout is to be in equilibrium, the set of equations (1), (2) and (3) must verify in each node and bar. To verify the geometrical compatibility equations (3) when $L_{i}^{\|}$is replaced by $L_{i}^{\|}(\alpha)=\alpha L_{i}^{\|}$, the cartesian components of all internal forces can be changed to

$F_{i}^{\| l}(\alpha)=F_{i}^{\| \mid}, \quad F_{i}^{\perp}(\alpha)=\frac{1}{\alpha} F_{i}^{\perp}$,

where the node equilibrium equations, (1), and (2), are satisfied in the new layout, as parallel components of external forces $\tilde{F}_{j}^{\|}$remain unchanged as internal are, and orthogonal components are null being the equations (2) for the new layout the original ones multiplied by $1 / \alpha$.

Therefore given a layout, with a given system of external forces that have not components in the orthogonal directions, the layout resulting from an affine transformation in depth of parameter $\alpha$ that equilbrates such set of forces has the same parallel components of the internal forces, but orthogonal components multiplied by $1 / \alpha$, i.e. $F_{i}^{||}(\alpha)=F_{i}^{\|}$and $F_{i}^{\perp}(\alpha)=\frac{1}{\alpha} F_{i}^{\perp}$, and has the same orthogonal components of bar lengths, but parallel bar lengths are multiplied by $\alpha$, i.e. $L_{i}^{\perp}(\alpha)=L_{i}^{\perp}$ and $L_{i}^{\|}(\alpha)=\alpha L_{i}^{\|}$.

From the definition of $\mathcal{V}=\sum_{i}\left|F_{i}^{\|} L_{i}^{\|}+F_{i}^{\perp} L_{i}^{\perp}\right|$ and taking into account that $\operatorname{sgn}\left(F_{i}^{\|} L_{i}^{\|}\right)=\operatorname{sgn}\left(F_{i}^{\perp} L_{i}^{\perp}\right)$ as result of compatibility equations (3), we can write $\mathcal{V}=\mathcal{V}^{\|}+\mathcal{V}^{\perp}$ where $\mathcal{V}^{\|}=\sum_{i}\left|F_{i}^{\|} L_{i}^{\|}\right|$and $\mathcal{V}^{\perp}=$ $\sum_{i}\left|F_{i}^{\perp} L_{i}^{\perp}\right|$. In the layout resulting from the affine transformation we obtain

$$
\begin{aligned}
& \mathcal{V}^{\|}(\alpha)=\sum_{i}\left|F_{i}^{\|} L_{i}^{\|}(\alpha)\right|=\alpha \sum_{i}\left|F_{i}^{\| \mid} L_{i}^{\|}\right|=\alpha \mathcal{V}_{1}^{\|} \\
& \mathcal{V}^{\perp}(\alpha)=\sum_{i}\left|F_{i}^{\perp}(\alpha) L_{i}^{\perp}\right|=\frac{1}{\alpha} \sum_{i}\left|F_{i}^{\perp} L_{i}^{\perp}\right|=\frac{1}{\alpha} \mathcal{V}_{1}^{\perp}
\end{aligned}
$$

where $\mathcal{V}_{1}^{\|}$and $\mathcal{V}_{1}^{\perp}$ stand for the parallel and orthogonal components of the stress volume of the original layout (corresponding to $\alpha=1$ ).

Therefore $\mathcal{V}^{\|}(\alpha)$ and $\mathcal{V}^{\perp}(\alpha)$ are such that

$$
\mathcal{V}^{\|}(\alpha) \mathcal{V}^{\perp}(\alpha)=\mathcal{V}_{1}^{\|} \mathcal{V}_{1}^{\perp}
$$

If we consider the set of layouts that balance a given set of external forces without orthogonal components, and can be obtained by an affine transformation of one given form with the objective of finding the depth which corresponds to a minimum stress volume we have to find the minimum of

$$
\mathcal{V}(\alpha)=\mathcal{V}^{\|}(\alpha)+\mathcal{V}^{\perp}(\alpha)
$$

subject to the constraint

$$
\mathcal{V}^{\|}(\alpha) \mathcal{V}^{\perp}(\alpha)=\mathcal{V}_{1}^{\|} \mathcal{V}_{1}^{\perp}
$$

This problem is equivalent to determine the rectangle of minimum (semi)perimeter between all rectangles of given area. The solution of this problem is well known and correspond to the rectangle of equal sides, i.e., the square.

In our case the solution corresponds to an optimal value of $\alpha$ : $\alpha^{*}$ for which

$\mathcal{V}^{\|}\left(\alpha^{*}\right)=\mathcal{V}^{\perp}\left(\alpha^{*}\right)=\sqrt{\mathcal{V}_{1}^{\|} \mathcal{V}_{1}^{\perp}}$

and $\alpha^{*}$ is given by

$\alpha^{*}=\frac{\mathcal{V}_{1}^{\perp}}{\mathcal{V}^{\perp}(\alpha)}=\frac{\mathcal{V}^{\|}(\alpha)}{\mathcal{V}_{1}^{\|}}=\sqrt{\frac{\mathcal{V}_{1}^{\perp}}{\mathcal{V}_{1}^{\|}}}$ 
If $d$ and $\lambda$ are respectively the depth and slenderness of the original layout, the depth and slenderness of the optimal layout are thus given by

$d^{*}=\alpha^{*} d, \quad \lambda^{*}=\frac{L}{d^{*}}=\frac{1}{\alpha^{*}} \frac{L}{d}=\frac{1}{\alpha^{*}} \lambda$

where $L$ is the unchanged reference span. (Q.E.D.).

Arocas's Theorem can be extended to systems of external forces where orthogonal components are not null. In this case original equations (2) read

$\tilde{F}_{j}^{\perp}=\sum_{i} F_{i}^{\perp}$

As before, with $F_{i}^{\perp}(\alpha)=\frac{1}{\alpha} F_{i}^{\perp}$, we must multiply equations $\left(2^{\prime}\right)$ by $1 / \alpha$ to keep equilibrium in the orthogonal direction. Hence the orthogonal components of the external forces must be multiplied by $1 / \alpha$. Thus the equilibrium of the new layout is maintained. This is the case for pinned archs vith vertical loads in which horizontal reactions must change inversely to depths in order to maintain the global moment equilibrium. The parallel and orthogonal components of the stress volume behave as before, and the optimal values are determined in the same way.

Returning to the set of parabolic arch problems and solutions - and beginning with problem in Authors' Table 1, third row, problem that can be stated as the reference case for all three shown there-, it can be seen then that optimal solution will have same stress volume in tie and hangers, each one with half of the arch stress volume that will have two equal components in the parallel — vertical - and orthogonal — horizontaldirections, each one corresponding to the counterparts in hangers or tie: we have in the optimum four equal components, two in tension $\mathcal{V}_{r}^{+\|}$(hangers) and $\mathcal{V}_{r}^{+\perp}$ (tie) and two in compression $\mathcal{V}_{r}^{-||}$and $\mathcal{V}_{r}^{-\perp}$ (both in the arch), having thus a total stress volume $\mathcal{V}_{r}=4 \mathcal{V}_{r}^{+\|}$.

If one changes the reference problem to a modified one - from third row to second row of Authors' Table 1 - by the elimination of the tie - and supporting thus the arch ends with oblique reactions - and one begins with the layout obtained from that one of the reference, one begins thus with a starting system with a total stress volume $\mathcal{V}=3 \mathcal{V}_{r}^{+\|}$but where horizontal and vertical components are not more equal, nor optimum. To get the optimum the vertical part must be reduced with an increase of the horizontal one, by reduction of depth. This case corresponds to the commented extension of Aroca's OS Theorem, and thus the optimal slenderness and stress volume can be deduced from those of the reference case as follows:
- the stress volume components of the starting solution are $\mathcal{V}^{+\|}, \mathcal{V}^{+\perp}, \mathcal{V}^{-\|}, \mathcal{V}^{-\perp}$, having

$-\mathcal{V}^{+\perp}=0$ (no tie)

$-\mathcal{V}^{-\|}=\mathcal{V}^{+\|}=\mathcal{V}_{r}^{+\|}$(load ascent via hangers equal to its descent via arch)

$-\mathcal{V}^{-\perp}=\mathcal{V}_{r}^{+\|}$ getting $\mathcal{V}^{\|}=2 \mathcal{V}^{\perp}$, and $\mathcal{V}=3 \mathcal{V}^{\perp}=3 \mathcal{V}_{r}^{+\|}$.

- using eq. (4) one gets $\alpha^{*}=\sqrt{\mathcal{V}^{\perp} / \mathcal{V} \|}=1 / \sqrt{2}$, having thus a reduction in depth of that magnitude.

- Optimum total stress volume can be computed: $\mathcal{V}^{*}=$ $2 \sqrt{\mathcal{V}^{\|} \mathcal{V} \perp}=2 \sqrt{\mathcal{V}_{r}^{+\|} 2 \mathcal{V}_{r}^{+\| \mid}}=2 \sqrt{2} \mathcal{V}_{r}^{+\|}$, where $\mathcal{V}_{r}^{+\|}$ comes out from the reference system's stress volume and $\mathcal{V}$ is the starting one after the elimination of the tie.

Thus, if reference problem depth was $\sqrt{3} / 4$ of span, and its stress volume divided by the product of total load times the span was $2 / \sqrt{3}$ (we call this value Michell number $\boldsymbol{\nu}$ of the layout, see Cervera Bravo et al (2014), and it can be written $\boldsymbol{\nu}=\sigma V_{T o t} / \hat{P}_{T} \hat{L}$, as Authors head their last column in Table 1$)$, the corresponding optimum values are thus $(\sqrt{3} / 4) / \sqrt{2}$ for the depht to span relation - the inverse of slenderness - and $2 \sqrt{2} \times(2 / \sqrt{3}) / 4=\sqrt{2} / \sqrt{3}$ for the Michell number, as Authors' Table 1 shows.

\section{Further notes}

In Writers' opinion, the engineering problem arises because of the magnitude, location and class of the useful loads are ever given. The first case of Authors' Table 1, where the researcher puts the load as his or her convenience is excepcional and of theoretical nature.

The single chord 16-Bay truss attributed to Hemp by the Authors was obtained by W.J. Supple (cited by Hemp, 1973:21).

Authors (p. 50) say that the pin-roller boundary condition "is very important in the engineering practice because it reduces the demand on the foundations". But from Writers' actual experience it can be said that the friction forces on the foundations are free of cost and ever present, so the roller boundary condition must be considered either as a theoretical artifact or as a practical method to eliminate thermal stresses. Frequently, the consideration of friction forces, when possible, reduces the cost of the structure without increasing the cost of the foundations (Cervera Bravo et al, 2014).

Authors say (p. 51) that "the tension force in the tie helps stabilize the arch from buckling", but whatever be the meaning of "stabilize" the same efect can be obtained from an horizontal reaction of the same magnitude, so it is not clear that any difference exist 
between tie or horizontal reaction of equal magnitude, putting aside the cost of the tie.

Some of the Authors' solutions are not restricted to equal stress in tension and in compression. In fact, when dealing with given external forces in equilibrium (i.e, with Maxwell problems, see Cervera Bravo et al, 2014:Definition 1) the optimal layout does not depend on the proportion of limiting stresses.

\section{Conclusions}

The present discussion has shown

- Similarities and differences between the solutions provided by Authors (Beghini et al, 2014) and Writers (Cervera Bravo et al, 2014).

- The relevancy and fruitfulness of dealing with optimization problems from the Maxwell and Michell approaches.

- The formulation of Aroca's Theorem on Optimum Slenderness, and its capability to explain layout and cost parameters in related structural problems.

\section{References}

Beghini A, Beghini LL, Baker WF (2014) On the layout of a least weight single span structure with uniform load. Struct Multidisc Optim 50:49-64, DOI 10.1007/ s00158-013-1030-6

Cervera Bravo J, Vázquez Espí C, Vázquez Espí M (2014) Two near-optimal layouts for truss-like bridge structures bearing uniform weight between supports. Journal of Structural Engineering 140:04013093-1 - 04013093-7, DOI 10.1061/(ASCE)ST.1493-541X. 0000905

Cervera J (2010) Concebir y analizar estructuras. UPM, Madrid, URL http://oa.upm.es/189, versión 3.0. http://oa.upm.es/3691

Fernández Cabo JL (1998) Estructura: tamaño, forma y proporción. PhD thesis, Escuela Técnica Superior de Arquitectura, Madrid, URL http://oa.upm.es/ 14488/

Hemp W (1973) Optimum structures. Clarendon, Oxford

Michell AGM (1904) The limits of economy of materials in frame-structures. Philosophical Magazine 8(47):589-597

Pichugin AV, Tyas A, Gilbert M (2012) On the optimality of Hemp's arch with vertical hangers. Structural and Multidisciplinary Optimization 46:17-25, DOI 10.1007/s00158-012-0769-5

Rozvany GIN, Prager W (1979) A new class of structural optimization problems: optimal archgrids.
Computer Methods in Applied Mechanics and Engineering 19(1):127-150

Sokół T (2014) Multiload-truss topology optimization using the adaptive ground structure approach. In: Łodygowski T, Rakowski J, Litewka P (eds) Recent Advances in Computational Mechanics, CRC Press, London, chap 2, pp 9-16

Vázquez Espí M, Cervera Bravo J (2011) On the solution of the three forces problem and its application in optimal designing of a class of symmetric plane frameworks of least weight. Struct Multidisc Optim 44:723-727, DOI 10.1007/s00158-011-0702-3 